\title{
Influence of Seismic Source Parameters on the Excitation Wavelet in Seismic Explorations Based on Numerical Simulation
}

\author{
Ruohua Qiu, ${ }^{1,2,3}$ Zhaoxing Dong $\mathbb{D}^{1},{ }^{1}$ Yanjun Qi, ${ }^{1}$ Yanhui Wang, ${ }^{4}$ Lidong Xie, ${ }^{1}$ \\ and Honghua Liu $^{2,3}$ \\ ${ }^{1}$ School of Mechanics and Civil Engineering, China University of Mining and Technology, Xuzhou 221116, China \\ ${ }^{2}$ Qingdao Geological Engineering Survey Institute, Qingdao 266100, China \\ ${ }^{3}$ Key Laboratory of Urban Geology and Underground Space Resources, \\ Shandong Provincial Bureau of Geology \& Mineral Resources, Qingdao 266100, China \\ ${ }^{4}$ Zhengzhou Urban Planning Design \& Survey Research Institute, Zhengzhou 450052, China \\ Correspondence should be addressed to Zhaoxing Dong; mtdzx41@163.com
}

Received 20 May 2020; Revised 3 July 2020; Accepted 6 July 2020; Published 24 July 2020

Academic Editor: Jia-wen Zhou

Copyright ( 2020 Ruohua Qiu et al. This is an open access article distributed under the Creative Commons Attribution License, which permits unrestricted use, distribution, and reproduction in any medium, provided the original work is properly cited.

\begin{abstract}
Explosives are the most common seismic source in seismic explorations, whose excitation effect is closely associated with their properties, weight, buried depth, and charging constitution. This study analyzes the influence of seismic source parameters on the features of the explosion wavelet. The explosion process of the seismic source in marine carbonate is numerically simulated with the finite element software LS-DYNA. We consider three parameters, including buried depth, charge weight, and decoupling coefficient. To verify the simulation outcomes, we also carry out field observations. The appropriate buried depth of the explosive should be determined according to stratum lithology. As the charge weight increases, low-frequency energy of the source wavelet increases rapidly. The optimum charge weight is $16-18 \mathrm{~kg}$, and the ideal charging structure is in a concentrated, short-column shape. Compared with the buried depth and charge weight, the decoupling coefficient shows more noticeable influence on the excitation effect of the source wavelet, and the optimum coefficient lies between 2.5 and 3 . The results of this study may provide reference data for designing explosion parameters in field seismic explorations.
\end{abstract}

\section{Introduction}

Seismic exploration has been the most important means for gas and oil exploration since 1920s. As a routine in onshore gas and oil seismic explorations, explosive seismic source serves as the first subsystem of the seismic source system. The selection of explosion parameters, such as detonation velocity, charge weight, coupling properties, and geometry coupling state, is important to recorded signal features, such as the waveform, amplitude, and frequency [1-4]. Selecting optimal excitation factors is a matter of excitability of highfrequency signals in the field of seismic exploration system engineering, and it is the basis for acquiring high-resolution data with a high signal-to-noise ratio $[5,6]$.

Source wavelet is the main form of seismic records, and, therefore, its calculation and analysis can be helpful for understanding the generation and propagation of seismic waves [7]. In a homogeneous rock stratum, the wavelet is influenced by the structure and physiomechanical characteristics of the rock; it is also affected by the excitation factors of the seismic source, such as the nature and charge weight of the explosives, the depth of the excitation well, and charging constitution [8-12]. To date, theoretical calculation methods for the relation between explosion parameters and the seismic source wavelet are limited in number [13, 14], and studies in this respect have long been heavily relying on field experiments $[8,12,15-17]$. However, field experiments cost high. In addition, in field experiments, the collected data are limited to the vibration signals of surface particles, which fail to completely describe the seismic wave field in the medium excited by explosion. Under such a condition, assistive techniques are always necessary. 
With the rapid development of explosion theories and computer science, numerical simulation has become an effective method for studying explosion waves and exploring the propagation laws of the waves. Artero-Guerrero et al. [18] constructed a numerical calculation model with LSDYNA (explicit, 3-dimensional, dynamic, and nonlinear finite element analysis software $[19,20])$ to analyze the influence of the mass and shape of explosives on the dynamics of the explosion wave. In their study, the charge weight was $10-20 \mathrm{~g}$ and five height-to-diameter ratios were designed. They found that given a certain mass of the explosive, the changes in the shape, pressure, and velocity of the explosion wave are all driven by the area perpendicular to the propagation of the explosive wave [18]. Wang et al. [21] performed separate numerical simulations with LSDYNA for the explosion processes of different explosives with different charge weights in limestone, sandstone, clays, and loess to investigate the relation between explosion parameters and the seismic source wavelet. They found that wavelets in rock and soil produced by explosions can be calculated based on the combination of numerical simulation, plastic-elastic boundary pressure curve fitting, and application of the spherical source model; in limestone and sandstone, a seismic source with a high detonation velocity explosive, a large charge weight, and water coupling is recommended, whereas in loess, a low detonation velocity explosive and a small charge weight is more suitable. These studies both lead to conclusions of practical significance. However, seismic source wavelet can be influenced by a variety of excitation factors in actual engineering practice, among which decoupling coefficient cannot be ignored. According to the formula of particle displacement in homogeneous elastic medium proposed by Sharpe [22], decoupling charging can change the cavity radius formed by the explosion as well as the pressure upon the inner wall of the cavity, which in turn influences the excitation effect on the source wavelet. Nevertheless, studies in this respect are rare.

Based on the aforementioned information, this study simulates the explosion process of the explosive seismic source in rock with LS-DYNA to investigate the influence of explosive buried depth, charge weight, and decoupling coefficient on the features of the seismic source wavelet. Based on comparative analysis, decoupling coefficient shows more noticeable influence on the seismic source wavelet than explosive buried depth and charge weight.

\section{Methodology}

2.1. Research Plan. The research plan of this study is illustrated in Figure 1.

2.2. Design of the Simulation Protocols. We use the buried depth, weight, and decoupling coefficient of the charge as variables for the numerical simulation experiment, and groupings are as follows:

Buried depths (10 levels): 17, 18, 19, 20, 22, 25, 30, 40, 55 , and $75 \mathrm{~m}$
Charge weights (10 levels): 10, 12, 14, 16, 18, 20, 25, 30, 40 , and $55 \mathrm{~kg}$

Decoupling coefficients (10 levels): 1.1, 1.2, 1.3, 1.45, $1.6,1.8,2.0,2.5,3.0$, and 4.0

The seismic source parameters are designed using the orthogonal experimental method [23] (Table 1), and, finally, $10^{3}=1000$ tests are required according to comprehensive collocations.

The randomization method [23] is adopted. According to L100 $\left(10^{3}\right)$ orthogonal optimization, we need to calculated 100 models in total to eradicate the influence of confounding factors on the exhibitions of the propagation features of the seismic source wavelet in the rock.

2.3. Material Parameters and Models. In this simulation experiment, the rock stratum excited by the seismic source is composed of marine carbonate, and its constitutive relation is described with a model for plastic kinematic hardening materials (*MAT_PLASTIC_KINEMATIC) [24]. This model is subject to a plastic kinematic model. Due to a large number of numerical simulation tests involved in this study, the adoption of this model can achieve high calculation efficiency; furthermore, this model can be used to realize the simulation of the explosion cavity by defining failure strain parameters [25-27]. The detailed parameters of the stratum are summarized in Table 2.

Fine sands are used as the coupling medium of the model, which is described with the MAT_SOID_AND_FORM model [24] (Table 3).

The explosive is described with the high-energy material model MAT_HIGH_EXPLOSIVE_BURN and Jones-Wilkins-Lee (JWL) state equation [24]. The JWL equation can precisely describe the pressure, volume, and energy features of the gaseous products during denotation, which is as follows [28]:

$$
p=A\left(1-\frac{\omega}{R_{1} V}\right) e^{-R_{1} V}+B\left(1-\frac{\omega}{R_{2} V}\right) e^{-R_{2} V}+\frac{\omega E_{0}}{V},
$$

where $p$ is the pressure of the denotation products, $V$ is the relative volume of the denotation products, $E_{0}$ is the initial inner energy density, and $A, B, R_{1}, R_{2}$, and $\omega$ are the constants determined by the experiment. The values of the explosive and state equation parameters are summarized in Table 4.

The geometry model, material models, and boundary conditions are input into the preprocessing module of the ANSYS software, and then grids are demarcated to establish a numerical calculation model. The model data are transformed into the $\mathrm{K}$ file with the calculation and analysis module of ANSYS, which is then introduced into LS-DYNA Solver for solution. LS-Prepost is used for postprocessing analysis of the calculated model. The completed model is able to realize the simulation of the influence laws of the seismic source parameters on the source wavelet in an infinite half space. In this experiment, the entire calculation model is cylindrical, whose upper boundary is a free surface, with the rest faces set to nonreflection boundaries 


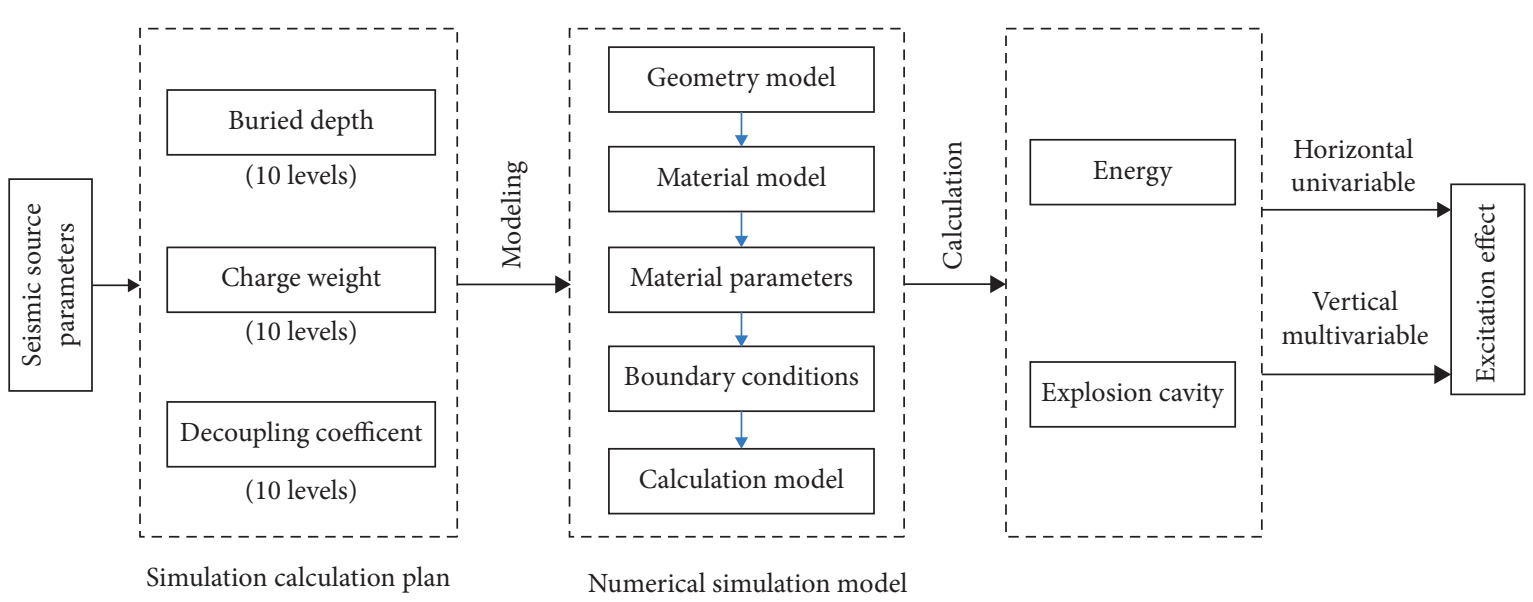

Figure 1: Research plan of this study.

TABLE 1: Orthogonal design of 10-level variables.

\begin{tabular}{|c|c|c|c|c|c|c|c|c|c|c|}
\hline \multirow{2}{*}{ Variable } & \multicolumn{10}{|c|}{ Level } \\
\hline & 1 & 2 & 3 & 4 & 5 & 6 & 7 & 8 & 9 & 10 \\
\hline Burie & A1 & A2 & A3 & A4 & A5 & A6 & A7 & A8 & A9 & A10 \\
\hline Charge weight & B1 & B2 & B3 & B4 & B5 & B6 & B7 & B8 & B9 & B10 \\
\hline $\begin{array}{l}\text { Decoupling } \\
\text { coefficient }\end{array}$ & $\mathrm{C} 1$ & $\mathrm{C} 2$ & $\mathrm{C} 3$ & $\mathrm{C} 4$ & C5 & C6 & C7 & $\mathrm{C} 8$ & C9 & $\mathrm{C}$ \\
\hline
\end{tabular}

TABLe 2: Parameters of the marine carbonate model.

\begin{tabular}{lcccc}
\hline$\rho\left(\mathrm{g} \cdot \mathrm{cm}^{-3}\right)$ & $E(\mathrm{GPa})$ & $\mu$ & $\theta$ & $V_{p}\left(\mathrm{~m} \cdot \mathrm{s}^{-1}\right)$ \\
\hline 2.694 & 8.51 & 0.363 & 0.0022 & 3218 \\
\hline
\end{tabular}

$\rho$, density; $E$, elastic modulus; $\mu$, Poisson's ratio of the rock; $\theta$, failure strain; $V_{p}$, velocity of the longitudinal waves of the rock.

(Figure 2(a)). The explosive is charged cylindrically and concentrated (Figure 2(b)). The height $h$ and diameter $d$ of the charge are calculated according to the explosive mass, with a height-diameter ratio of 4 . The diameter of the blast hole $D$ is calculated based on $d$ and the decoupling coefficient $D / d$, and the depth of the blast hole $H$ based on $h$ and the buried depth $l$. Separate 3-dimensional numerical simulation calculation models are established (Figure 2(c)).

2.4. Field Measurement. Field tests on the buried depth and charge weight of the seismic source are conducted in a marine carbonate outcropping area in Bazhong, Sichuan province, China. The seismic data are collected from the ground for excitation analysis. The collection conditions for ground signals included the following: trace interval, $30 \mathrm{~m}$; radiodetector, 2 buried square combinations of 18 SJ-40 radiodetectors, with a combination base distance of $4 \mathrm{~m}$; sampling rate, $1 \mathrm{~ms}$; and preamplifier gain, $42 \mathrm{~dB}$.

The working conditions are as follows:

Condition 1: charge weight, $10 \mathrm{~kg}$, and buried depth, $15 \mathrm{~m}, 18 \mathrm{~m}, 20 \mathrm{~m}, 22 \mathrm{~m}, 24 \mathrm{~m}, 26 \mathrm{~m}$, and $28 \mathrm{~m}$, respectively
Condition 2: buried depth, $20 \mathrm{~m}$, and charge weight, 12, $14,16,18$, and $20 \mathrm{~kg}$, respectively

\section{Results and Discussion}

3.1. Field Measurements. Figure 3 shows the energygram according to the seismic records obtained at different buried depths. The energy is powerful at a buried depth of $15-20 \mathrm{~m}$, after which it is weakened to some extent.

Figure 4 shows the energy and signal-to-noise ratios according to the seismic records under different charge weights. Under a charge weight of $16 \mathrm{~kg}$, the reflected wave possesses the most powerful energy and the highest signalto-noise ratio, showing the most satisfactory excitation effect.

\subsection{Simulation Results}

3.2.1. Attenuation of the Seismic Source Wavelet. For cylindrical concentrated charges, the wave front of the seismic source wavelet can be considered as a spherical surface. Based on the viscoelastic medium wave equation, the energy of the wavelet is a function of transmission distance [29]. Therefore, we use the relative distance to the excitation source to constitute the $x$-axis of the coordinate and set 10 data collection points for each model (Table 5).

The test data are reviewed, and 30 models that are distorted due to relatively great influence of model boundaries (buried depths at 40,55, and $75 \mathrm{~m}$ ) are excluded. In addition, to ensure that the simulation results are as close to actual values as possible, we put every 10 models into one group, in which a weighted mean is used for the same collection point to describe the particle velocity (Figure 5).

As shown in Figure 5, all curves show similar attenuation trends but without overlapping, which indicates that the excitation modes have influence on the propagation of the source wavelet and that the energy transmitted by the source wavelet can be regulated by changing explosion parameters. In Figure 5, the relative distance of each data collection point to the excitation source point $r$ is $x-H$. When $r$ is $>60 \mathrm{~m}$, the slope of the attenuation curve $k$ approaches 0 . This finding 


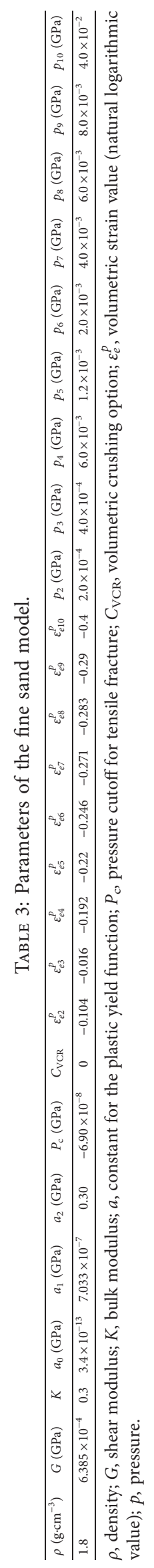


TABLE 4: Parameters of the explosive in the numerically stimulation experiment.

\begin{tabular}{lcccccccc}
\hline$\rho\left(\mathrm{kg} / \mathrm{m}^{3}\right)$ & $D(\mathrm{~m} / \mathrm{s})$ & $P_{\mathrm{CJ}}(\mathrm{GPa})$ & $A(\mathrm{GPa})$ & $B(\mathrm{GPa})$ & $R_{1}$ & $R_{2}$ & $\omega$ & $E_{0}\left(\mathrm{~J} / \mathrm{m}^{3}\right)$ \\
\hline 1630 & 6930 & 27 & 374 & 7.33 & 4.15 & 0.95 & 0.3 & $7 \times 10^{9}$ \\
\hline
\end{tabular}

$\rho$, density; $D$, detonation velocity; $P_{\mathrm{CJ}}$, Chapman-Jouguet pressure.

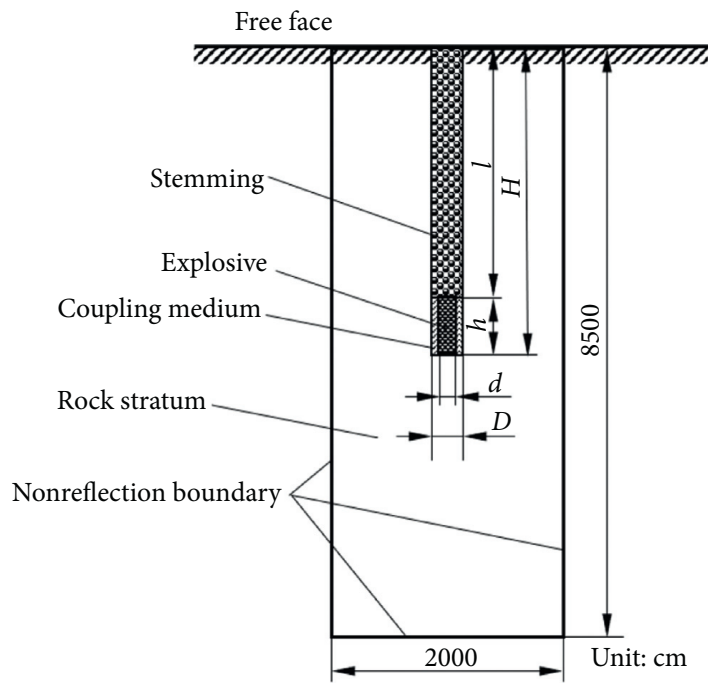

(a)

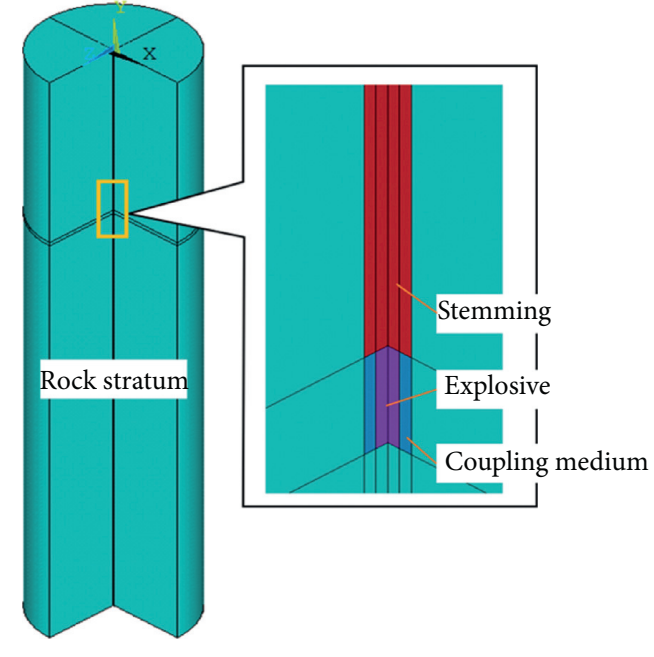

(b)

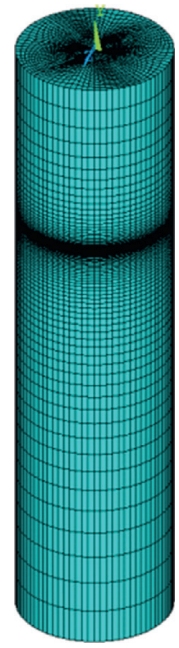

(c)

FIgURE 2: The numerical simulation model. (a) Size of the model. (b) 3/4 of the model and the charging constitution. (c) The numerical simulation model.

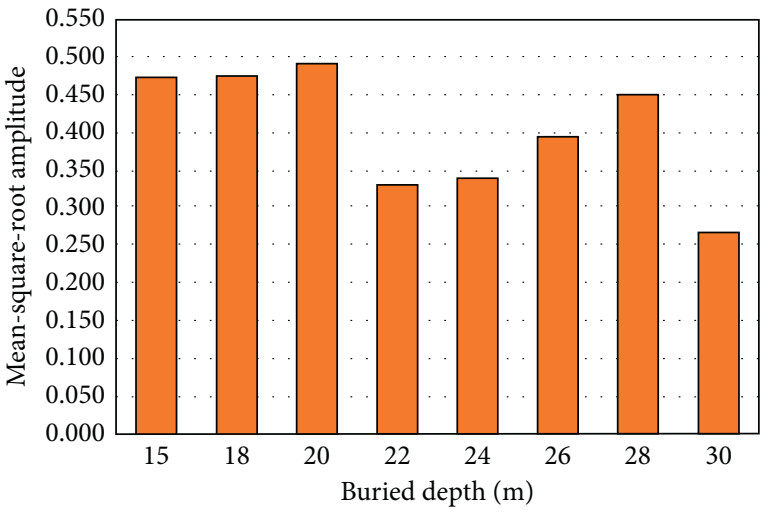

FIGURE 3: The energygram according to the seismic recorded obtained at different buried depths of the charge.

indicates that the energy of the seismic source wavelet attenuates sharply within $60 \mathrm{~m}$ in marine carbonate and becomes stable when $r$ is $>60 \mathrm{~m}$. The transmitted energy attenuates exponentially with the extension of the relative distance [30].

3.2.2. Energy Analysis. In this study, the depth size of the models is $85 \mathrm{~m}$. According to the calculated energy attenuation results, we remove the 40 models with a wavelet transmission distance $<25 \mathrm{~m}$ (85 minus 60) (i.e., buried depths at $30 \mathrm{~m}, 40 \mathrm{~m}, 55 \mathrm{~m}$, and $75 \mathrm{~m}$ ) to avoid possible outcome distortions due to boundary settings. At the same distant point ( $84 \mathrm{~m}$ below the Earth's surface) of the remaining 60 models, energy $<100 \mathrm{~Hz}$ is statistically calculated. The outcomes of the polar analysis of variance are summarized in Table 6.

As shown in Table 6, the decoupling coefficient is significant at the level of $p=0.98$ and the charge weight is significant at the level of $p=0.70$. However, buried depth remains insignificant even at a level of $p=0.50$. Range reflects the variation amplitude of the tested index when variables change. The results of this study show $D_{\mathrm{C}}>D_{\mathrm{B}}>D_{\mathrm{A}}$, which indicates that decoupling coefficient is the most influencing factor for transmitted energy, followed by the charge weight and then buried depth.

Based on the field observations and the outcomes of the polar analysis of variance, we perform extra tests on decoupling coefficients $(5.0,6.0,8.0,12$, and 18), with the buried depth of $20 \mathrm{~m}$ and the charge weight of $16 \mathrm{~kg}$. The influence of different explosion parameters on low-frequency energy of the seismic source wavelet is shown in Figure 6.

As shown in Figure 6, low-frequency energy does not noticeably increase with the increase in the buried depth of the charge. The reason under this phenomenon may be as follows. Compared with the depth of oil reserves, the variation range of the buried depth of the explosive is quite small. Theoretically, explosives of the same mass possess identical chemical energy, and, therefore, energy of the seismic source wavelets produced by them is definite [19]. If the energy released after explosion can be completely transmitted to the rock, rather than being dissipated in rock throwing and air blast forming, it is 


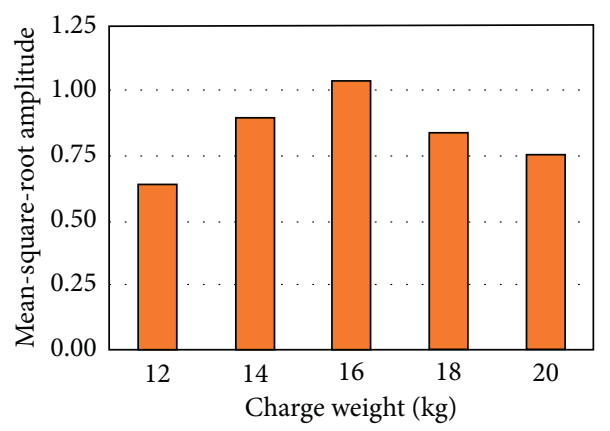

(a)

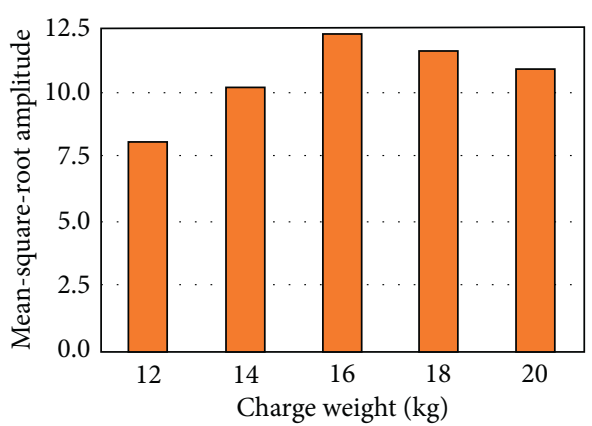

(b)

FIgURE 4: Seismic energy (a) and signal-to-noise ratios (b) under different charge weights.

TABLE 5: Distribution of data collection points.

\begin{tabular}{lccccccccccc}
\hline Collection point & P1 & P2 & P3 & P4 & P5 & P6 & P7 & P8 & P9 & P10 \\
\hline Relative coefficient $a_{i}$ & 0.1 & 0.129 & 0.167 & 0.215 & 0.278 & 0.36 & 0.464 & 0.599 & 0.774 & 1 \\
\hline Relative distance & & \multicolumn{1}{c}{$r=(8500-H) \times a_{i}$} \\
\hline
\end{tabular}

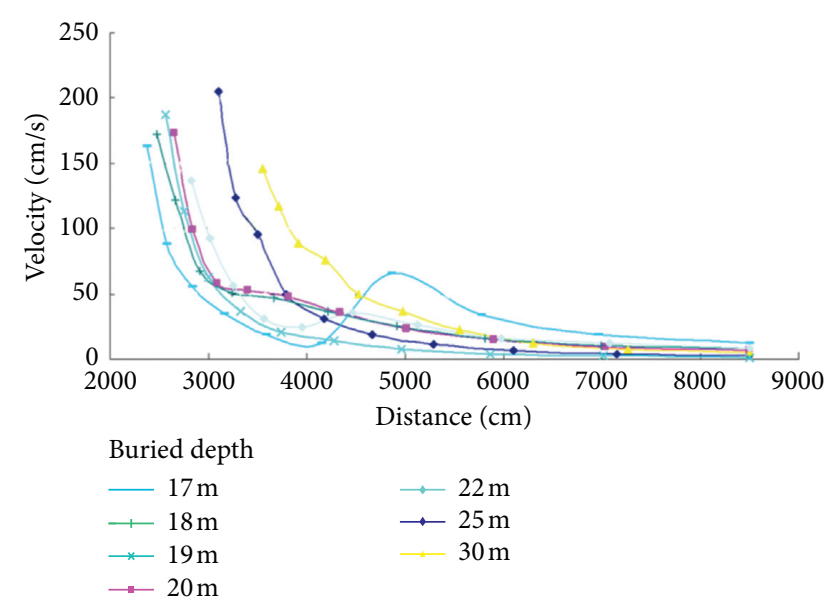

FIGURE 5: Attenuation principle of the seismic source wavelet in marine carbonate.

indicated that the buried depth of the explosive is sufficient in actual practice. However, according to the field observations in this study, the energy of the seismic source wavelet varies noticeably when the buried depth exceeds $20 \mathrm{~m}$. Presumably, this phenomenon is due to heterogeneity of the actual stratum. According to Wang et al. [21], the seismic wavelets vary according to the properties of the rock even when the same explosive is used. Therefore, in field seismic explorations, rock properties should be taken into consideration in determining the buried depth of the charge.

Figure 6 also shows that low-frequency energy increases rapidly with the increase in the charge weight, and the optimum charge weight is $16 \mathrm{~kg}$, which is consistent with the field observation. According to studies on the explosions of hemispherical and flat explosives $[19,31,32]$, the diameter of the charge rather than the charge weight serves as the leading factor for an increase in downward-transmitted energy; the larger the diameter of the charge column is, the more the transmitted energy will be. In our study, the height and diameter ratio of the charge is $4: 1$. With the increase in the charge weight, the charge diameter increases accordingly.

In addition, as shown in Figure 6, with the gradual increase in the decoupling coefficient, low-frequency energy increases gradually to the peak, which is then followed by a rapid decrease. The coefficient corresponded by the peak is 4 . The variation trend of low-frequency energy according to the decoupling coefficient obtained in this study is similar to those reported by Triviño et al. [11], Wang et al. [21], and Yao et al. [33]. However, the exact decoupling coefficient corresponded by the peak in our study is different from those in the literature $[11,22,33]$. This inconsistency is presumably due to the differences in the selection of other explosion parameters.

According to explosion theories, the range of explosion cavity is 3-7 times the radius of the charge [34]. Therefore, when the decoupling coefficient is $>8$, the blast has already weakened a lot in the coupling medium, which cannot break marine carbonate any longer. At this moment, low-frequency energy is transmitted directly into the rock. In the meantime, the blast forms a cavity in the low-density and -stiffness medium. Because of the existence of the cavity, the energy loss through the cavity is less than that through marine carbonate. Under such a condition, low-frequency energy increases gradually.

3.2.3. Analysis of Explosion Cavity. In this study, the model for marine carbonate is $*$ MAT_PLASTIC_KINEMATIC. This model possesses self-attached failure criteria, which can be defined by assigning a value to the failure strain of the parameters $[19,20]$. In this study, the value assigned to the failure strain of the marine carbonate model $\theta$ is 0.0022 (Table 2); that is, units with a strain $>0.0022$ in the model will be removed and replaced by a cavity. In this way, the cavity formation in marine carbonate under explosion load can be exhibited [35]. Based on 
TABLE 6: Outcomes of the polar analysis of variance (energy unit, $10^{-6} \mathrm{~J}$ ).

\begin{tabular}{lccc}
\hline Index & Buried depth $A(\mathrm{~m})$ & Charge weight $B(\mathrm{~kg})$ & Decoupling coefficient $C$ \\
\hline Range $D_{n}(n=A, B, C)$ & 47933 & 112660 & 257736 \\
Deviation sum $S_{n}$ & 22321313936 & 71939729597 & 369129801271 \\
Degree of freedom $f_{n}$ & 9 & 5 & 5 \\
Variance $V_{n}$ & 2480145993 & 14387945919 & 73825960254 \\
Variance ratio $F_{n}$ & 0.28 & 1.65 & 8.46 \\
$p$ & $F_{p}(9,5)$ & $F_{p}(5,5)$ & $F_{p}(5,5)$ \\
0.9995 & 36.3093 & 39.7194 & 39.7194 \\
0.999 & 27.2445 & 29.7524 & 29.7524 \\
0.9975 & 18.5367 & 20.1783 & 20.1783 \\
0.995 & 13.7716 & 14.9396 & 14.9396 \\
0.99 & 10.1578 & 10.967 & 10.967 \\
0.98 & 7.4152 & 7.9529 & 7.9529 \\
0.975 & 6.6811 & 7.1464 & 7.1464 \\
0.95 & 4.7725 & 5.0503 & 5.0503 \\
0.9 & 3.31628 & 3.45298 & 3.45298 \\
0.85 & 2.62677 & 2.69799 & 2.69799 \\
0.8 & 2.19628 & 2.22752 & 2.22752 \\
0.75 & 1.89106 & 1.89466 & 1.89466 \\
0.7 & 1.65789 & 1.64098 & 1.64098 \\
0.65 & 1.47069 & 1.43783 & 1.43783 \\
0.6 & 1.31486 & 1.26923 & 1.26923 \\
0.55 & 1.18151 & 1.12542 & 1.12542 \\
0.5 & 1.06478 & 1 & 1 \\
Significance & & $(0.70)$ & $(0.98)$ \\
\hline
\end{tabular}

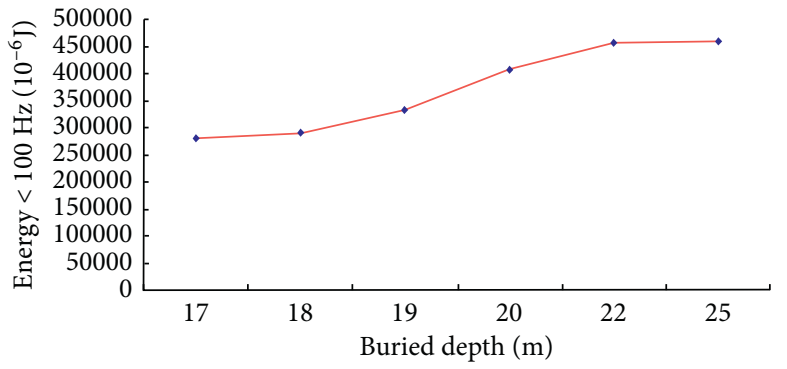

(a)

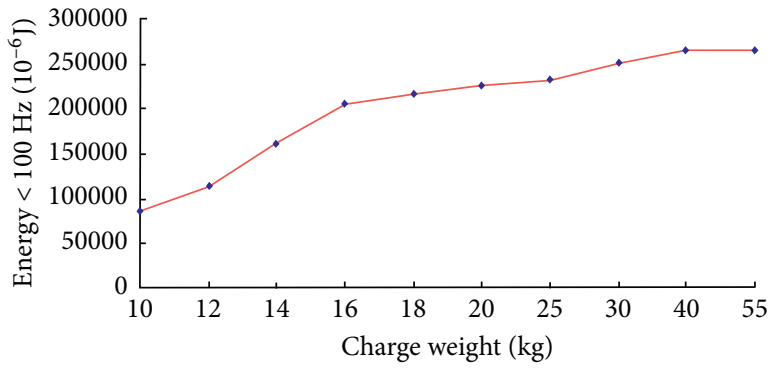

(b)

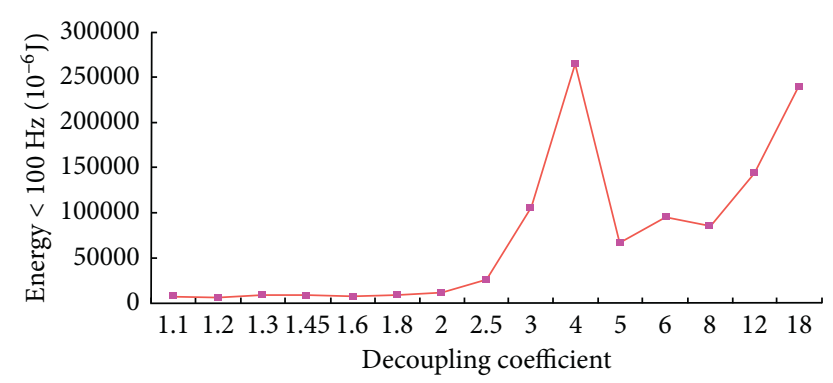

(c)

FIGURE 6: Variations in low-frequency energy of the seismic source wavelet according to different explosion parameters. (a) Buried depth. (b) Charge weight. (c) Decoupling coefficient. Data are the means of the values at the same collection point of 10 models.

the results of the experiment, the explosion cavity in marine carbonate formed after explosion is elliptical. The extension diameter of the cavity is measured in the direction along the diameter of the charge, and Figure 7 is obtained.

As shown in Figure 7, with the increase in the decoupling coefficient, the diameter of the cavity gradually increases, and the variation trends under different charge weights are similar.
When the decoupling coefficient value is small, the ratio between the diameter of the cavity and that of the explosive cylinder under a small charge weight is larger than that under a large charge weight. When the decoupling coefficient lies between 2.5 and 3 , the ratio under a large charge weight is larger than that under a small charge weight. These findings indicate that at a small decoupling coefficient, energy loss in 


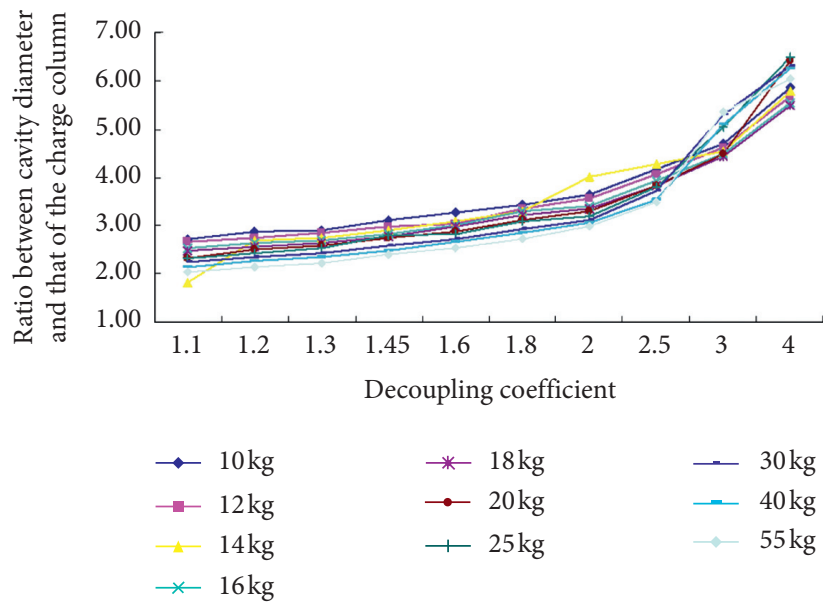

(a)

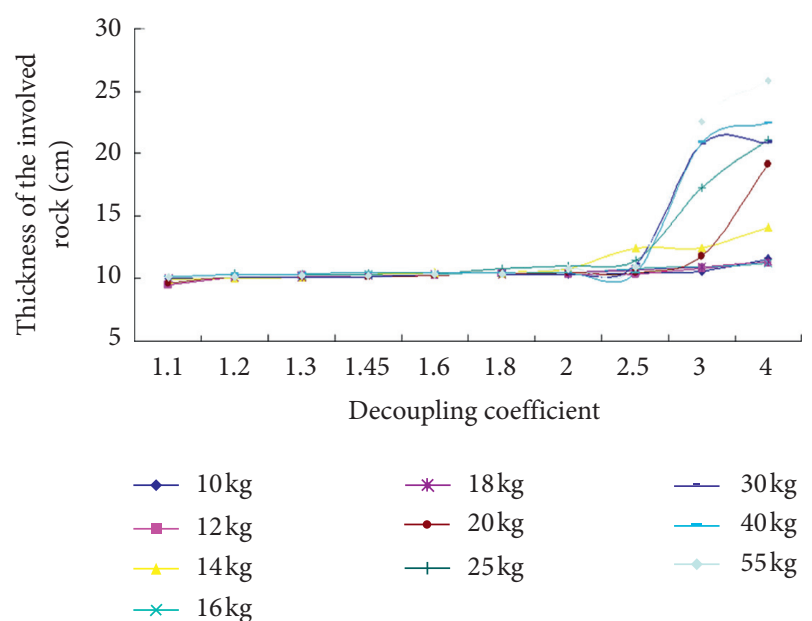

(b)

Figure 7: Statistics of the diameter of the cavity.

breaking the rock is large; the diameter of the cavity is correlated with the diameter of the explosive cylinder, whereas the contribution of the charge weight to the diameter of the cavity is small.

When the decoupling coefficient is $<3$, the thickness of the rock involved by the cavity basically remains unvaried. This is because the wave impedance of the coupling medium needs to be between the rock and the explosive to maintain effective energy transmission. The rock is far stiffer than the coupling medium, and, therefore, the energy consumed in breaking the rock is much larger than that in forming a cavity. Under the same charge weight, the existence of the coupling medium reduces the thickness of the involved rock. In the meantime, it absolutely increases the diameter of the cavity. Because the bottom surface of the charge cylinder is a plane, an increase in the diameter of the cavity increases the contact area of downward transmission of energy. Therefore, an increase in the decoupling coefficient gives rise to a gradual increase in energy that is transmitted downwards. However, when the decoupling coefficient reaches 4 , the thickness of the involved rock increases abruptly. At this moment, energy used for breaking the rock increases, and that for downward transmission drops. This finding is also evidenced by that based on the range analysis in this study. Therefore, based on the consideration of the energy loss due to rock breaking, a satisfactory decoupling coefficient should be $<3$.

\section{Conclusions}

In this study, we investigate the influence of explosive buried depth, charge weight, and decoupling coefficient on the seismic source wavelet using the numerical simulation method. We draw the following conclusions:

(1) From the perspective of well depth and charge weight, a deeper well and a larger charge weight do not indicate a more satisfactory excitation effect. In field seismic explorations, the properties of rock should be taken into consideration in determining the buried depth of the charge. The optimum charge weight is $16-18 \mathrm{~kg}$. Low-column concentrated charging can achieve a satisfactory excitation effect, which is manifested by powerful downwardtransmitted energy and a high signal-to-noise ratio of the wavelet.

(2) Decoupling coefficient is the most influencing factor for the seismic source wavelet, among the investigated parameters, whose variations show noticeable influence on the excitation effect on the wavelet. An increase in the decoupling coefficient increases the volume of the explosion cavity, as well as the contact area of the downward surface wave. For the interest of low-frequency energy transfer, the decoupling coefficient should be 2.5-3.

In this study, we analyze the influence principles of the buried depth, charge weight, and decoupling coefficient on the explosion wavelet and compare their contributions to the influence. Decoupling coefficient has more noticeable influence on the source wavelet compared with the buried depth of the explosive and the charge weight. According to the results, we propose explosion parameters suitable for marine carbonate, which can serve as a guide for designing ideal explosion parameters in field seismic explorations. However, source wavelet can be affected by a variety of factors, and the number of the investigated factors in this study is limited. In the future, comparative studies on more factors should be conducted to identify and optimize dominant factors for excitation wavelet. In addition, due to the limitations of the mountains and road conditions in the field test site, transportation of different types of drilling tools, as well as the supporting equipment of the tools, to verify the simulation outcomes related to the decoupling coefficients is difficult to complete. Therefore, the optimal 
decoupling coefficient obtained in this study needs to be validated by field experiments in the future.

\section{Data Availability}

All data for analysis in this study are included within the article.

\section{Conflicts of Interest}

The authors declare no conflicts of interest.

\section{References}

[1] S. H. Qian and T. S. Li, "The research on the explosive mechanism and excitation condition of dynamite source," Geophysical Prospecting for Petroleum, vol. 37, pp. 1-14, 1998.

[2] L. D. Xie, Z. X. Dong, Y. J. Qi, R. H. Qiu, and Q. He, "Vibration failure of young low-temperature concrete shaft linings caused by blasting excavation," Advances in Civil Engineering, vol. 2019, Article ID 5343618, 10 pages, 2019.

[3] R. C. Selley and S. A. Sonnenberg, Elements of Petroleum Geology, Academic Press, Cambridge, MA, USA, 2015.

[4] Y. Lan, C. Y. Sun, and Y. F. Yan, "On relevance between explosive parameters and seismic wavelets," Oil Geophysical Prospecting, vol. 51, no. 5, pp. 13-19, 2016.

[5] W. F. Pan, W. W. Zhao, X. S. Meng et al., "The choice of optimum charge and coupling condition in high-resolution seismic exploration," Oil Geophysical Prospecting, vol. 4, pp. 443-451, 2000.

[6] S. M. Gandhi and B. C. Sarkar, Essentials of Mineral Exploration and Evaluation, Elsevier, Amsterdam, Netherlands, 2016.

[7] N. Ricker, "The form and nature of seismic waves and the structure of seismograms," Geophysics, vol. 5, no. 4, pp. 348-366, 1940.

[8] H. R. Nicholls, "Coupling explosive energy to rock," Geophysics, vol. 27, no. 3, pp. 305-316, 1962.

[9] J. R. Grant, A. T. Spathis, and D. P. Blair, "An investigation of the influence of charge length upon blast vibrations," in Proceedings of the 6th ISRM Congress. International Society for Rock Mechanics and Rock Engineering, Montreal, Canada, September 1987.

[10] J. W. Cheng, H. M. Gu, and Z. Y. Xu, "Simulation of explosion source wavelet in the actual formation," Chinese Journal of Engineering Geophysics, vol. 6, pp. 529-534, 2009.

[11] L. F. Triviño, B. Mohanty, and B. Milkereit, "Seismic waveforms from explosive sources located in boreholes and initiated in different directions," Journal of Applied Geophysics, vol. 87, pp. 81-93, 2012.

[12] C.-H. Bai, J. Chen, B. Zhang, and Z.-Q. Wang, "Effect of explosive sources on the elastic wave field of explosions in soils," Defence Science Journal, vol. 63, no. 4, pp. 376-380, 2013.

[13] A. Ziolkowski, "Determination of the signature of a dynamite source using source scaling, part 1: theory," Geophysics, vol. 58, no. 8, pp. 1174-1182, 1993.

[14] D. Blair, "Seismic radiation from an explosive column," Geophysics, vol. 75, no. 1, pp. E55-E65, 2010.

[15] P. N. S. O'Brien, "Some experiments concerning the primary seismic pulse," Geophysical Prospecting, vol. 17, no. 4, pp. 511-547, 1969.

[16] X. Cai, W. Su, and H. Xu, "Further understanding of the characteristic impedance coupling of explosives to rock," in
Proceedings of the SEG Technical Program Expanded Abstracts 2010, pp. 137-141, Tulsa, OK, USA, 2010.

[17] A. Stroujkova, M. Leidig, and J. L. Bonner, "Effect of the detonation velocity of explosives on seismic radiation," Bulletin of the Seismological Society of America, vol. 105, no. 2A, pp. 599-611, 2015.

[18] J. Artero-Guerrero, J. Pernas-Sánchez, and F. Teixeira-Dias, "Blast wave dynamics: the influence of the shape of the explosive," Journal of Hazardous Materials, vol. 331, pp. 189199, 2017.

[19] LSTC, LS-DYNA Theoretical Manual, Livermore Software Technology Corporation (LSTC), Livermore, CA, USA, 2006.

[20] LSTC, LS-DYNA Keyword User's Manual, Vol. I, Livermore Software Technology Corporation (LSTC), Livermore, CA, USA, 2007.

[21] Y. M. Wang, G. Tian, and L. Y. Yu, "The effect of explosion parameters on seismic source wavelet calculation and its characteristics," Journal of Applied Geophysics, vol. 145, pp. 50-58, 2017.

[22] J. A. Sharpe, "The production of elastic waves by explosion pressures. I. Theory and empirical field observations," Geophysics, vol. 7, no. 2, pp. 144-154, 1942.

[23] K. M. Ramachandran and C. P. Tsokos, Mathematical Statistics with Applications in $R$, Academic Press, Cambridge, MA, USA, 2nd edition, 2015.

[24] LSTC, LS-DYNA Keyword User's Manual, Volume II: Material Models, Livermore Software Technology Corporation (LSTC), Livermore, CA, USA, 2013.

[25] R. S. Yang, C. X. Ding, Y. B. Wang et al., “Action-effect study of medium under loading of explosion stress wave and explosion gas," Chinese Journal of Rock Mechanics and Engineering, vol. S2, pp. 3501-3506, 2016, in Chinese.

[26] N. Jiang, C. Zhou, S. Lu, and Z. Zhang, "Propagation and prediction of blasting vibration on slope in an open pit during underground mining," Tunnelling and Underground Space Technology, vol. 70, pp. 409-421, 2017.

[27] R. Yang, C. Ding, L. Yang, Z. Lei, and C. Zheng, "Study of decoupled charge blasting based on high-speed digital image correlation method," Tunnelling and Underground Space Technology, vol. 83, pp. 51-59, 2019.

[28] P. S. Westine, J. J. Kulesz, P. A. Cox et al., Explosion Hazards and Evaluation, Elsevier, Amsterdam, Netherlands, 1983.

[29] A. M. Abo-Zena, "Radiation from a finite cylindrical explosive source," Geophysics, vol. 42, no. 7, pp. 1384-1393, 1977.

[30] Y. J. Qi, Z. X. Dong, and H. W. Jing, "Experimental study on energy attenuation of limestone seismic wave," New Progress of Research on the Coordinated Development of Civil Engineering and Environment, vol. 12, pp. 138-144, 2010.

[31] A. Lefrançois, S. Chapelle, S. Pauly et al., Tremendous Increase of the Blast Effect above HE Plates Characterized by Small Scale Experiments, Xth MABS, Israel, 2010.

[32] J. Mespoulet, F. Plassard, P. Hereil et al., "Influence of HE shape on blast profile," in Proceedings of the 8th European LSDYNA Users Conference, pp. 2-12, Strasbourg, France, 2011.

[33] Z. Yao, Z. Du, X. Wang et al., "Seismic acquisition techniques and their application in hill areas with thick loess, western foreland of ordos basin," Petroleum Exploration and Development, vol. 34, p. 406, 2007.

[34] P. A. Persson, R. Holmberg, and J. Lee, Rock Blasting and Explosives Engineering, CRC Press, Boca Raton, FL, USA, 2018.

[35] D. Y. Shi, Y. C. Li, and J. M. Zhang, Explicit Dynamic Analysis Basing on ANSYS/LS_DYNA 8.1, Tsinghua University Press, Beijing, China, 2004. 